\title{
Sustained Release of Naltrexone from Poly(N-Isopropylacrylamide) Microgels
}

\author{
ANNA-LENA KJØNIKSEN, ${ }^{1,2}$ MARIA TERESA CALEJO,${ }^{1,3}$ KAIZHENG ZHU, ${ }^{4}$ ANA MARIA S. CARDOSO, \\ MARIA C. PEDROSO DE LIMA, ${ }^{5,6}$ AMÁLIA S. JURADO,, ${ }^{5,6}$ BO NYSTRÖM, ${ }^{4}$ SVERRE ARNE SANDE ${ }^{1}$ \\ ${ }^{1}$ Department of Pharmacy, School of Pharmacy, University of Oslo, Blindern, N-0316 Oslo, Norway \\ ${ }^{2}$ Faculty of Engineering, Østfold University College, N-1757 Halden, Norway \\ ${ }^{3}$ Department of Electronics and Communications Engineering, Tampere University of Technology, FI-33101 Tampere, Finland \\ ${ }^{4}$ Department of Chemistry, University of Oslo, Blindern, N-0315 Oslo, Norway \\ ${ }^{5} \mathrm{CNC}$ - Centre for Neuroscience and Cell Biology, University of Coimbra, Coimbra, Portugal \\ ${ }^{6}$ Department of Life Sciences, University of Coimbra, Coimbra, Portugal
}

Received 12 September 2013; revised 17 October 2013; accepted 18 October 2013

Published online 11 November 2013 in Wiley Online Library (wileyonlinelibrary.com). DOI 10.1002 /jps.23780

\begin{abstract}
The release of the opioid antagonist naltrexone from neutral poly( $\mathrm{N}$-isopropylacrylamide) (PNIPAAM) microgels and negatively charged PNIPAAM microgels containing acrylic acid groups (PNIPAAM-CO-PAA) has been studied at various microgel and drug concentrations. The release curves were found to be well represented by the Weibull equation. The release rates were observed to be dependent on the microgel concentration. At most conditions, the release from the charged microgels was slower than for the neutral microgels. In addition, the charged microgels exhibited a release lag time, which was dependent on the microgel concentration. No significant lag time could be observed for the neutral microgels. Increasing the naltrexone concentration did not significantly affect the release rates from the neutral microgels, but the release from the charged microgels became faster. The microgels did not exhibit any significant cytotoxic effect on HeLa cells at the tested concentrations. (c) 2013 Wiley Periodicals, Inc. and the American Pharmacists Association J Pharm Sci 103:227-234, 2014
\end{abstract}

Keywords: controlled release; drug delivery systems; macromolecular drug delivery; microencapsulation; microparticles; polymeric drug delivery systems

\section{INTRODUCTION}

Naltrexone (Fig. 1) is an opioid antagonist, and has been used to treat heroin and alcohol addiction. However, oral naltrexone formulations have drawbacks such as patient noncompliance, ${ }^{1}$ fluctuating plasma levels, ${ }^{2}$ and the extensive first-pass metabolism of naltrexone. ${ }^{3}$ Injectable depot formulations that can give a sustained release of naltrexone over an extended period of time could prevent the problems encountered with oral dosage forms. Several systems such as poly(L-lactide) microspheres, ${ }^{4,5}$ poly lactide-co-glycolide (PLGA) microspheres, ${ }^{6}$ poly(D,L-lactideco-glycolide) microspheres, ${ }^{7}$ polyethylene glycol-graft-methyl methacrylate crosslinked nanoparticles, ${ }^{8}$ and nanoparticles produced from a blend of $\operatorname{poly}(N$-isopropylacrylamideacrylamide-vinylpyrrolidone) and $\mathrm{PLGA}^{9}$ have been suggested for sustained release dosage forms for naltrexone. There is however still need for improving the release profile and drug loading capacity of the systems. Utilizing new kinds of thermosensitive in situ gelling microgels might therefore be of interest.

The release of a drug that is encapsulated into micro- or nanoparticles is dependent on a number of factors such as particle size ${ }^{10-13}$ drug size, ${ }^{14,15}$ the degree of swelling of the particles, ${ }^{16-18}$ erosion of the particles, ${ }^{19}$ and associative interactions between the drug and the particles, for example, hy-

Correspondence to: Anna-Lena Kjøniksen (Telephone: +47-69104095; Fax: +47-69215002; E-mail: anna.l.kjoniksen@hiof.no)

Journal of Pharmaceutical Sciences, Vol. 103, 227-234 (2014)

(C) 2013 Wiley Periodicals, Inc. and the American Pharmacists Association drophobic interactions, ${ }^{15}$ hydrogen bonds, ${ }^{20,21}$ or electrostatic interactions. $^{14}$

Nano- and microparticles that are formed using thermosensitive polymers with a lower critical solution temperature (LCST) have a swollen structure at temperatures below the transition temperature and a more compact (collapsed) structure at higher temperatures. This effect can be utilized both to increase the loading capacity and modulate the release of a drug from the particles. Increased loading rate and capacity may be expected for drug loading in the swollen state, whereas the drug release is often found to be slower when the systems are heated to temperatures above the LCST of the particles due to reduced porosity. This phenomenon has been observed for several different systems such as: core-shell nanoparticles containing a poly(L-lactic acid) core and a poly $(N$ isopropylacrylamide) (PNIPAAM) thermosensitive shell, ${ }^{22}$ alginate-hydroxypropylcellulose microbeads, ${ }^{23}$ poly $(N$-vinyl caprolactam $)$ nanoparticles, ${ }^{24}$ poly $(N$-isopropylacrylamide-cocaprolactam) microspheres, ${ }^{25}$ and PNIPAAM microspheres grafted with poly(itaconic acid) groups. ${ }^{14}$ Interestingly, microspheres of elastin-like polypeptides (ELP) exhibited faster release rates above the LCST due to the opening of micropores in the microspheres by the contraction of the thermosensitive ELP molecules. ${ }^{26}$ Faster release rates above the LCST was also observed for chitosan-g-poly( $N$-vinylcaprolactam $)$ and chitosan- $g$ PNIPAAM nanoparticles. ${ }^{20,21}$ This was explained by the ability of the drug to form hydrogen bonds with the nanoparticles at temperatures below the LCST. This capacity was lost above the LCST, and hence faster release rates are promoted. 


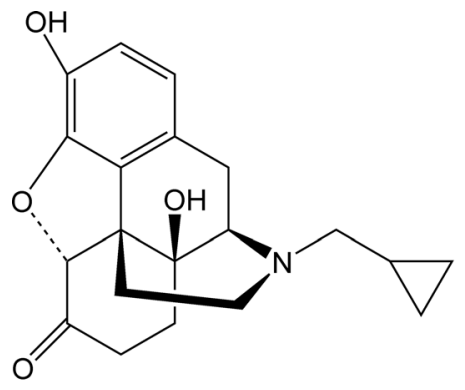

Naltrexone<smiles>CC(C)CC(C)C(=O)NC(C)C</smiles>

PNIPAAM

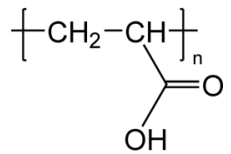

PAA

Figure 1. Chemical structure of naltrexone, PNIPAAM, and PAA.

For $\operatorname{poly}(N$-isopropylacrylamide-co-acrylamide $)$ and $\operatorname{poly}(N$ isopropylacrylamide-co- $N$-hydroxymethyl acrylamide) microspheres, it was found that even though the drug release is slower above the LCST, there is an enhanced release of drug for a short period when the temperature is increased from below to above the LCST. ${ }^{15,27}$ This effect was thought to be due to drug molecules that are dissolved in the solvent inside the swollen microspheres. When the samples are heated up, these drug molecules are squeezed out together with the solvent during the collapse of the network. Similar effects have also been observed for other thermoresponsive systems. ${ }^{28}$

In this study, we have investigated PNIPAAM microgels as a sustained drug delivery vehicle for naltrexone. The microgel concentrations utilized in this study are quite high, and it is not possible to measure their sizes using light scattering methods due to multiple scattering. However, previous studies of the same microgels at lower concentrations show that the microgels are swollen and have an open structure at low temperatures. ${ }^{29}$ As the temperature is increased the microgels shrink, and at temperatures above the LCST of PNIPAAM (ca. $32^{\circ} \mathrm{C}$ ) large aggregates with a compact structure are formed.

The drug is loaded into the microgels at a low temperature. At these conditions, the microgels are swollen in the solvent, and this open structure should make it easier for the drug to diffuse into the microgels. The microgel suspensions have a low viscosity and can be easily administrated to the patient through injection. When the samples are heated up to body temperature, the microgels collapse and they also aggregate into larger structures. ${ }^{29} \mathrm{~A}$ schematic illustration of a possible mechanism for loading of naltrexone into the PNIPAAM microgels is depicted in Figure 2. The conjecture is that both the more compact structure and the formation of larger aggregates at $37^{\circ} \mathrm{C}$ should slow down the diffusion of naltrexone out of the microgels. As naltrexone contains hydrophobic domains, associative interactions between the drug and the PNIPAAM microgels could also result in slower drug release rates.

Naltrexone is positively charged at physiological $\mathrm{pH} .{ }^{30} \mathrm{We}$ have therefore also studied PNIPAAM microgels containing $1 \mathrm{~mol} \%$ acrylic acid groups to see if the electrostatic interactions between the drug and the oppositely charged microgels can slow down the release rates. At low microgel concentrations, this low charge density was observed to have little effect on the contraction and aggregation behavior of the microgels in water. ${ }^{29}$ Any changes in the observed release profiles are therefore mainly expected to be due to electrostatic interactions between the drug and the microgels.

As for any proposed drug delivery system, the toxicity of the formulation is of vital importance. We have therefore also conducted in vitro cytotoxicity studies on the microgels at the considered concentrations.

\section{MATERIALS AND METHODS}

The naltrexone (USPH1453504) was purchased from VWR International (West Chester, Pennsylvania, USA), and the chemicals used for making the buffer solution were purchased from Sigma-Aldrich (St. Louis, Missouri, USA).
Low temperatures

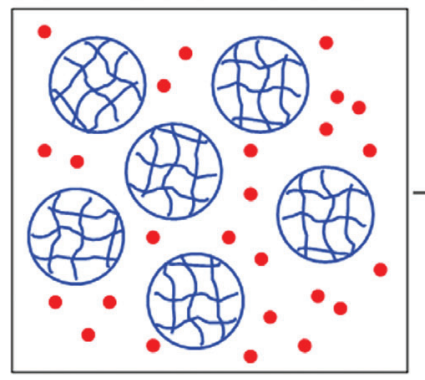

The microgels are mixed with the drug.

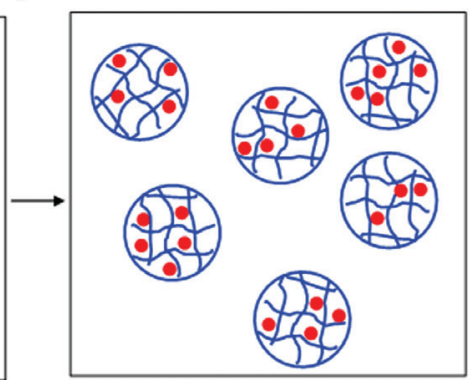

At low temperatures, the drug diffuses into the swollen microgels.

Figure 2. Schematic illustration of the proposed loading of naltrexone into the microgels. 


\section{Preparation of PNIPAAM Microgels}

Negatively charged PNIPAAM microgels (MG-04) containing $1 \mathrm{~mol} \%$ acrylic acid groups (PNIPAAM-co-PAA) and neutral PNIPAAM microgels (without acrylic acid groups; MG-06) were synthesized as described previously. ${ }^{29}$ The structures of PNIPAAM and PAA are shown in Figure 1.

\section{Sample Preparation}

A PBS was used for the preparation of the samples for the dissolution tests. The buffer contained $16.8 \mathrm{mM} \mathrm{Na}_{2} \mathrm{HPO}_{4}, 1.4 \mathrm{mM}$ $\mathrm{KH}_{2} \mathrm{PO}_{4}, 137 \mathrm{mM} \mathrm{NaCl}$, and $3.1 \mathrm{mM}$ sodium azide. The $\mathrm{pH}$ of the buffer was adjusted with acetic acid to 7.4.

For the release experiments, the microgels were dispersed in PBS at the desired concentrations (0-4 wt \%), and stirred for at least $24 \mathrm{~h}$. Afterward, the samples were placed in the fridge (ca. $4{ }^{\circ} \mathrm{C}$ ) for at least another $24 \mathrm{~h}$ to ensure homogeneous suspensions. Naltrexone $(0.02-0.08 \mathrm{wt} \%)$ was loaded into the microgels by dissolving it directly in the microgel suspensions. The samples were then stirred for at least $24 \mathrm{~h}$, and then placed in the fridge for at least $48 \mathrm{~h}$ before the release experiments were commenced. The concentrations given for naltrexone and the microgel suspensions are of the prepared suspensions before the samples were diluted down by the initiation of the release experiments.

\section{In Vitro Cytotoxicity Assay of the Microgels}

HeLa cells (human epithelial cervical carcinoma cell line) were maintained in culture at $37^{\circ} \mathrm{C}$, under $5 \% \mathrm{CO}_{2}$, in Dulbecco's modified Eagle's medium-high glucose (DMEM-HG; Sigma), supplemented with $10 \%(\mathrm{v} / \mathrm{v})$ heat inactivated fetal bovine serum (Sigma), penicillin (100 U/mL), and streptomycin (100 $\mu \mathrm{g} / \mathrm{mL}$ ). HeLa cells grown in monolayer were detached by treatment with $0.25 \%$ trypsin solution (Sigma).

The cell viability in the presence of the microgels was investigated by the modified Alamar Blue assay. This method, which has been extensively used to assess the viability and/or proliferation of mammalian cells, employs a redox indicator whose color (and fluorescence) changes in response to cell metabolic activity, the blue nonfluorescent oxidized form (resazurin) originating a pink and fluorescent molecule (resorufin) upon reduction. ${ }^{31}$ Briefly, HeLa cells were seeded at a cell density of 50,000 cells/well in 48 -well plates and incubated at $37^{\circ} \mathrm{C}$ for $24 \mathrm{~h}$. The microgels were suspended in Opti-MEM (reduced serum) medium at different concentrations and were added to the cells. The plates were left to incubate at $37^{\circ} \mathrm{C}$ for $48 \mathrm{~h}$. The medium was replaced with $300 \mu \mathrm{L}$ of $10 \%(\mathrm{v} / \mathrm{v})$ Alamar Blue (Sigma) dye in DMEM cell culture medium and the plates were further incubated for $45 \mathrm{~min}$ at $37^{\circ} \mathrm{C}$. An aliquot of the supernatant $(150 \mu \mathrm{L})$ was collected from each well and transferred to 96 -well plates. The absorbance at $570 \mathrm{~nm}\left(A_{570}\right)$ and $600 \mathrm{~nm}$ $\left(A_{600}\right)$ (information provided by the supplier) was measured in a SPECTRAmax PLUS 384 spectrophotometer. Cell viability was calculated according to the equation:

$$
\text { Cell viability }(\%)=\frac{\left(A_{570}-A_{600}\right) \text { of treated cells }}{\left(A_{570}-A_{600}\right) \text { of control cells }} \times 100
$$

\section{Release Experiments}

The release experiments were conducted utilizing a SOTAX USP Apparatus four flow through cell dissolution apparatus at $37^{\circ} \mathrm{C} .2 .5 \mathrm{~mL}$ of the samples was placed in the flow-through cells (which were filled with $1 \mathrm{~mm}$ glass beads to avoid turbulence and ensure a laminar flow of the buffer through the cells ${ }^{32}$ and left to equilibrate at $37^{\circ} \mathrm{C}$ for $10 \mathrm{~min}$ before the release experiments were initiated. During the release studies, $22.5 \mathrm{~mL}$ of PBS (giving a total volume of $25 \mathrm{~mL}$ ) was continuously pumped through the flow through cells at a flow rate of $4 \mathrm{~mL} / \mathrm{min}$. The microgels were prevented from escaping the cells by a combination of two filters: a membrane of regenerated cellulose with a $1 \mathrm{kDa}$ cutoff (Z-MEM-AQU-425N from Postnova) followed by a dialysis membrane (Spectra/Por 6 with a cutoff of $8000 \mathrm{Da}$ ). The buffer was circulating in a closed system, and the circuit was connected to a spectrophotometer (Pharmacia LKB - Ultrospec III), which detected the released naltrexone by measuring the absorbance at a wavelength of $282 \mathrm{~nm}$ every $2 \mathrm{nd} \min$ for a total release time of $5 \mathrm{~h}$.

The threshold concentration for departure from sink conditions for naltrexone is approximately $0.5 \mathrm{wt} \%,{ }^{33}$ and accordingly all release experiments have been conducted well within sink conditions.

\section{Evaluation of the Release Rates}

The release data have been fitted to the Weibull equation ${ }^{34,35}$ :

$$
\frac{M_{t}}{M_{\infty}}=1-\exp \left[-\left(\frac{t-t_{0}}{\tau_{\mathrm{d}}}\right)^{\beta}\right]
$$

where $M_{t}$ is the amount of drug released at the time $t, M_{\infty}$ is the maximum amount of drug released at infinite time, $t_{0}$ is the dissolution lag time, the rate constant $\tau_{\mathrm{d}}$ is the time after $t_{0}$ when $63.2 \%$ of the drug has been released, and $\beta$ is the shape parameter of the release curve.

For the neutral microgels, $t_{0}$ was always found to be zero within the experimental errors. The release curves for these microgels were therefore fitted using $t_{0} \equiv 0$ to reduce the number of fitting parameters.

The release curves for the samples where no lag time was observed were also fitted to an equation where an extra term was added to Eq. (2) to accommodate for a possible burst release effect. However, this resulted in very poor fits, and the burst release doses were always found to be zero within the experimental errors. This indicates that the systems do not exhibit any burst release, and the extra term were therefore not used in the analyses of the data.

The Weibull equation is often found to give a good fit to experimental drug release data over the whole experimental time scale. ${ }^{36-40}$ In spite of its statistical origin, the Weibull model can be used to gain information about the physiochemical aspects behind the release profile. Both experimental data ${ }^{10-13}$ and Monte Carlo simulations ${ }^{41,42}$ show that the drug is released slower from larger particles, that is, $\tau_{\mathrm{d}}$ becomes higher as the particle size is increased. The reduction of the specific leak surface (surface area/volume) of the particles with increasing size plays an important role in this phenomenon. ${ }^{41,42}$

The shape parameter, $\beta$, may be related to the release mechanism. ${ }^{36,43}$ Monte Carlo simulations also suggests that the value of $\beta$ depends both on the size of the particles ( $\beta$ decreases when the particles becomes larger), on the shape of the particles, and on the ability of the drug molecules to move inside the particle matrix ${ }^{42}$ For Fickian diffusion, an increase of $\beta$ should indicate less disorder inside the particles. ${ }^{36}$ 


\section{RESULTS AND DISCUSSION}

\section{In Vitro Cytotoxicity}

Because the microgels are intended for drug delivery purposes, it is important that they do not exhibit any significant cytotoxic effect. It has previously been found that PNIPAAM nanoparticles did not exhibit any cytotoxicity at concentrations up to about 1 wt $\%$, whereas NIPAAM monomers were found to be toxic even at low concentrations. ${ }^{44}$ We have tested the two microgels used in this study on HeLa cells using the same microgel concentrations as for the release experiments. The cell viability for different concentrations of the two microgels is displayed in Figure 3. Comparing the viability of cells incubated with the microgels to that of control cells, taken as $100 \%$, it is noticeable that microgels did not exhibit any significant cytotoxicity even at relatively high concentrations. At the lower concentrations assayed, the neutral PNIPAAM microgels apparently induced an increase of cell viability relative to control cells. To correctly interpret the meaning of this effect, it is necessary to take into account the principle that underlies the technique used to assess cell viability. The Alamar Blue assay uses a dye as an oxidation-reduction indicator, whose reduction occurs inside the cells ${ }^{31}$ and involves reductases and the mitochondrial electron chain. ${ }^{45}$ Therefore, compounds that promote an increase of nonviable cells, which have no metabolic capacity, will induce lower reduction of the dye. On the contrary, agents or conditions that enhance cell metabolic activity and/or promote higher rates of cell growth will induce higher levels of dye reduction. Both these phenomena (improvement of cell metabolic activity and increase of cell proliferation) can be on the basis of the high values of cell viability $(>100 \%)$ found at low concentrations of neutral PNIPAAM microgels. This effect has been observed before for other systems, and was attributed to the surface properties of the systems. ${ }^{46}$ It is also possible that the microgels act as a scaffold, thereby increasing the proliferation rates of the cells. ${ }^{47}$

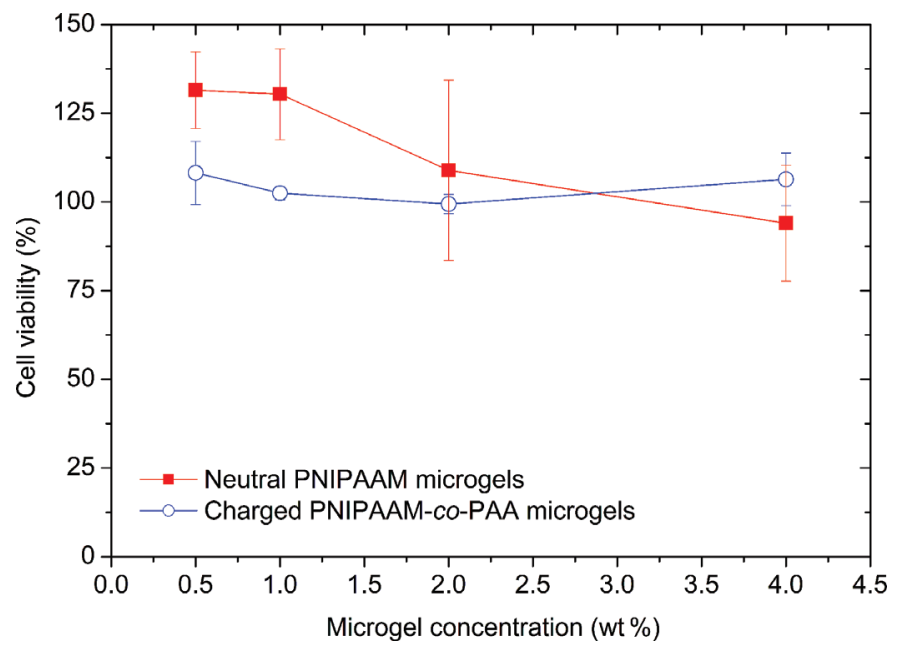

Figure 3. Effects of microgels on the viability of HeLa cells with increasing concentrations at $37^{\circ} \mathrm{C}$. Values of cell viability, measured by the Alamar Blue assay as described in Materials and Methods, are expressed as a percentage of the untreated cells (control) and are means \pm standard deviation obtained from triplicates in at least three independent experiments.

\section{In Vitro Release}

The release profiles of $0.02 \mathrm{wt} \%$ naltrexone from suspensions of the two microgels in phosphate buffer $(\mathrm{pH} 7.4)$ at $37^{\circ} \mathrm{C}$ are depicted in Figure 4 . When a buffer solution containing naltrexone in the absence of any microgel suspension ( $0 \mathrm{wt} \%$ microgel) is used, the time it takes for the system to detect $100 \%$ of the naltrexone is very short (ca. $5 \mathrm{~min}$ ). This indicates that the detection delay time of the experimental setup is fast. For both microgel systems, the release rate is observed to decrease as the microgel concentration is raised. Interestingly, a release lag time is evident for the charged microgels but not for the neutral microgels. This suggests that the charges modulate the way in which the naltrexone is released from the microgels. To quantify the variations in the release profiles, the curves have been fitted with the Weibull equation (Eq. 2). The solid lines in Figure 4 represent the fitted curves, and this illustrates that the data is well represented by the Weibull equation. The good fitting of the experimental data with the Weibull equation suggests that practically all of the naltrexone is encapsulated within the particles leaving an insignificant amount of unencapsulated naltrexone in the suspension. Accordingly, naltrexone is probably attached to the microgels through associative interactions, as drug molecules that are just dissolved in the solvent inside the swollen microgels are expected to be pushed out of the microgels when they are heated up above the LCST. ${ }^{15,27}$

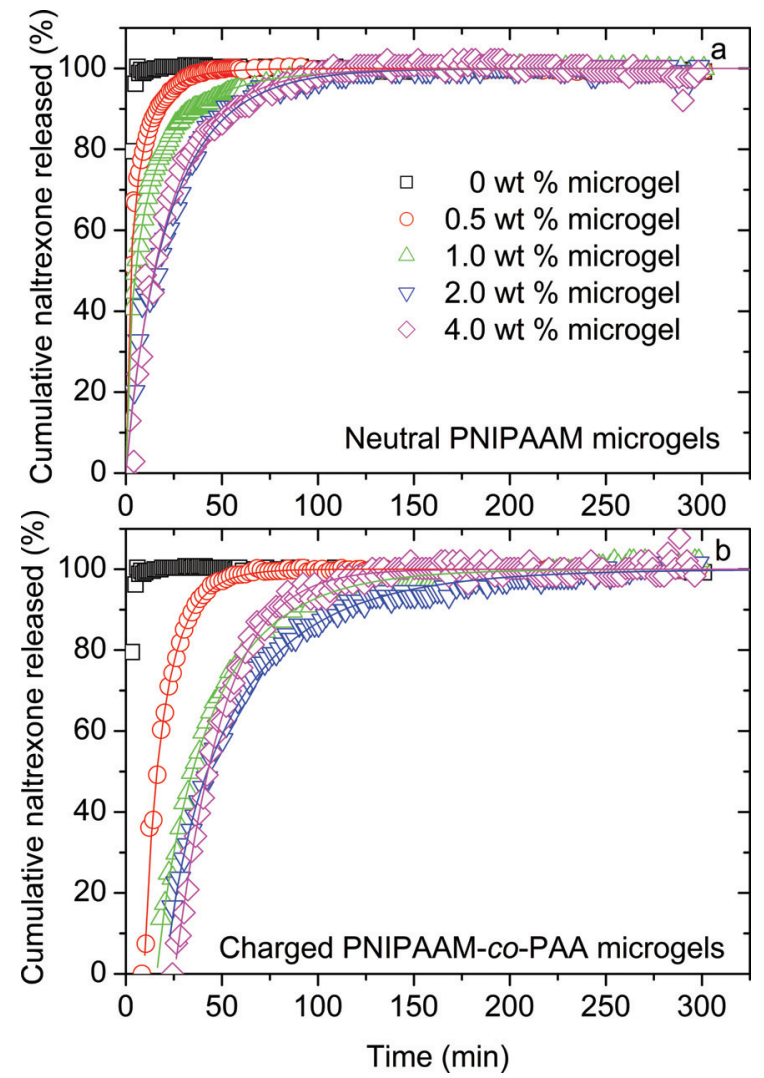

Figure 4. In vitro release profiles of naltrexone $(0.02 \mathrm{wt} \%)$ from the two microgels in buffered suspensions ( $\mathrm{pH} 7.4$ ) at various microgel concentrations at $37^{\circ} \mathrm{C}$. The sample denoted " $0 \mathrm{wt} \%$ microgel" consists of naltrexone dissolved in PBS in the absence of microgel. The lines are fitted with the aid of Eq. (2). 

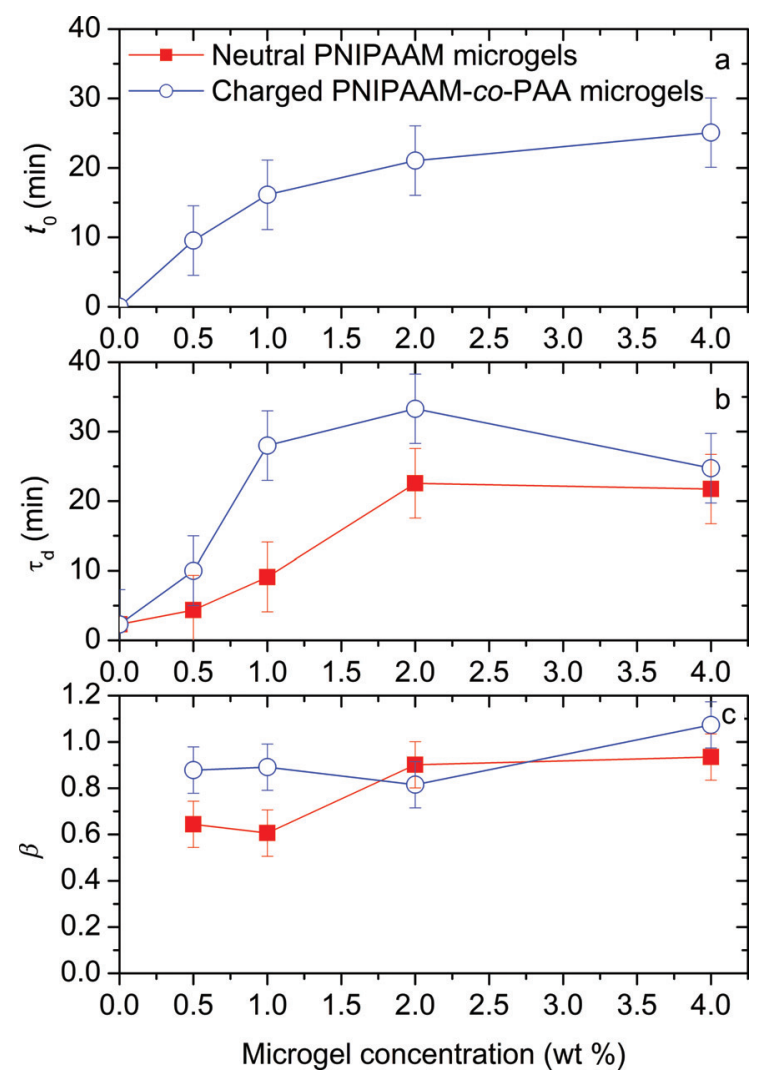

Figure 5. The effect of microgel concentration for the two microgels on (a) the lag time $\left(t_{0}\right)$, (b) the rate constant $\left(\tau_{\mathrm{d}}\right)$, and (c) the shape parameter $(\beta)$ for samples containing $0.02 \mathrm{wt} \%$ of naltrexone.

Although the neutral microgels did not exhibit any release lag time, a significant lag time was observed for the charged microgels (see Fig. 5a). For most release studies from uncoated micro/nanoparticles, no release lag time is observed, and a burst release effect is much more common. ${ }^{48}$ However, a significant lag time has been detected for some micro- and nanoparticle systems. ${ }^{49}$ In this study, the lag times were attributed to a combination of a very low adsorption of the probe molecules onto the surface of the particles and migration of the probe from the inner regions of the particles to their surface. Accordingly, the lag times observed for the charged microgels indicate that practically all of the naltrexone is encapsulated well within the microgels.

The absence of a lag time for the neutral microgels suggests that the charged groups play a vital role in inducing the lag times. Naltrexone has $\mathrm{p} K_{\mathrm{a}}$ values of about 8.1 (proton on $\mathrm{N}$ ) and 9.5 (phenolic proton) at $37^{\circ} \mathrm{C},{ }^{30}$ and can therefore be expected to be positively charged at the considered $\mathrm{pH}$ of 7.4. Considering the relatively high salinity of the buffer, some screening of electrostatic interactions in the system is expected. Unfortunately, the microgel suspensions are too turbid for zeta potential determination at the considered conditions. However, zeta potential measurements of other charged particles at corresponding salinities show that even though the addition of salt reduces the zeta potentials, the particles can still have a significant charge at these conditions. ${ }^{50-52}$ It is therefore reasonable to assume that there are attractive electrostatic interactions between the positively charged drug and the negatively charged PAA groups that are distributed throughout the charged microgels.
Taşdelen et al. ${ }^{14}$ found that the addition of charged poly(itaconic acid) groups to PNIPAAM microspheres slowed down the drug release from the particles due to electrostatic interactions with oppositely charged drugs. Although no release lag time was observed for this system, it was found that the charged groups prevented a significant part of the drugs to be released from the particles due to specific binding of the drugs to the dissociated acid groups. In our case, all naltrexone was released from the microgels, but the electrostatic interactions between the drug and the oppositely charged microgels causes a lag time before any drug is released from the particles. The lag time is increasing when the microgel concentration is raised. The reason for this will be discussed below.

Figure $5 \mathrm{~b}$ shows the rate constant $\left(\tau_{\mathrm{d}}\right)$, corresponding to the time (after the initial lag time) it takes to release $63.2 \%$ of the encapsulated naltrexone (see Eq. 2). It can be observed from Figure $5 \mathrm{~b}$ that an increase in the microgel concentration results in a slower release of the drug. As increasing the microgel concentration can cause enhanced aggregation of the microgels into larger particles, ${ }^{29}$ the slower release rates might be due to the larger sizes (see Evaluation of the Release Rates above). At high microgel concentrations, $t_{\mathrm{d}}$ is no longer dependent on the amount of microgel present. This could be because the specific leak surface becomes less size dependent for large particles.

It is obvious from Figure $5 \mathrm{~b}$ that the release of naltrexone is slower for the negatively charged microgels than for the neutral ones. Slower release rates might be caused by larger or more compact particles, or by stronger interactions between the drug and the microgel. However, at very low polymer concentrations, the addition of this small amount of charges to the PNIPAAM microgels had little effect on contraction and aggregation of the microgel suspensions. ${ }^{29}$ Even if the charges should influence the aggregation and contraction behavior of the microgels at the higher microgel concentrations and higher ionic strength utilized in this study, the charges are expected to reduce the tendency to form large aggregates, and in addition the repulsive electrostatic forces should also make the microgels less compact. It is therefore unlikely that the slower release rates observed for the charged microgels are due to larger particles or a more tight structure within the charged microgels. Accordingly, it is reasonable to assume that the slower release rates are caused by the attractive electrostatic interactions between the drug and the oppositely charged microgels.

The shape parameter, $\beta$, is plotted as a function of microgel concentration in Figure 5c. $\beta$ values in the range 0.39-0.69 indicate diffusion in a fractal or disordered substrate different from a percolation cluster, values in the range $0.69-0.75$ suggest diffusion in normal Euclidian space, whereas values between 0.75 and 1 are indicative for diffusion in normal Euclidian space with a contribution of another release mechanism. ${ }^{36}$ The $\beta$ values for the charged microgels are all in the range between 0.75 and 1 . This seems reasonable as the charges will prevent the microgels from contracting too much; thereby leaving a network that is open enough for the naltrexone to diffuse through as a normal Euclidian space. This $\beta$ range also indicates that there is an additional release mechanism, which is in agreement with the electrostatic interactions between the drug and the oppositely charged microgels.

At low microgel concentrations, the neutral microgel has $\beta$ values around 0.69 . Accordingly, the release mechanism is either due to diffusion in a fractal or disordered substrate or 
in normal Euclidian space. At high microgel concentrations, the $\beta$ values of the neutral microgels are in the region 0.75 1. This suggests diffusion in normal Euclidian space with a contribution of another release mechanism, which is probably caused by hydrophobic interactions between naltrexone and the microgels.

For both microgel systems, the $\beta$ values seem to be somewhat higher when the microgel concentration is raised. Increasing the microgel concentration can induce aggregation and thereby the formation of larger particles. ${ }^{29}$ However, increased particle size is expected to decrease the values of $\beta .^{42} \beta$ is also dependent on the intermolecular distances within the matrix, and will increase when the intermolecular distances become larger, ${ }^{42}$ that is, when the matrix structure is more open. This might explain why the charged microgels, which are expected to be more swollen due to electrostatic repulsions, have higher $\beta$ values than the neutral microgels at low microgel concentrations. It is possible that an increased microgel concentration causes enhanced associations between the polymer chains inside the particles. These associations may lead to the formation of a more inhomogeneous intraparticle network with a more open structure ${ }^{53,54}$ which might cause the observed increase in $\beta$. Another possible explanation for the increased $\beta$ values at high microgel concentrations is that the release profile is influenced by enhanced hydrophobic interactions between naltrexone and the microgels.

Because the naltrexone seemed to be completely encapsulated within the microgels when a naltrexone concentration of $0.02 \mathrm{wt} \%$ was used, it was also interesting to examine whether the microgels were capable of encapsulating even higher amounts of the drug. We therefore carried out some experiments where the microgel concentration was kept constant at $1.0 \mathrm{wt} \%$, and the naltrexone concentration was increased from 0.02 to $0.08 \mathrm{wt} \%$. Figure 6 shows the drug release profiles for the two microgel suspensions at three different naltrexone concentrations. As for the previous results, the curves were fitted to the Weibull equation (Eq. 2), and the fitted curves give a good representation of the measured data. There is a clear change in the release profile when the naltrexone concentration is raised above $0.02 \mathrm{wt} \%$ for the charged microgel (Fig. 6b), whereas the effect of naltrexone concentration is less obvious for the neutral microgels (Fig. 6a).

Interestingly, the release lag time of the charged microgels is independent on the naltrexone concentration (see Fig. 7a). We have already established that the lag time is due to electrostatic interactions, and that increasing the microgel concentration causes longer lag times. As the naltrexone concentration does not affect the lag time, the ratio between naltrexone and the charged groups on the microgels is not the determining factor. The microgel concentration dependency (Fig. 5a) therefore indicates that the lag time is dependent on the characteristics of the microgel aggregates. This seems reasonable as the microgels are expected to form larger aggregates when the microgel concentration is increased. The lag time should be dependent on the time it takes the drug to diffuse from its location inside the particles and out to the surface. Therefore, the longer lag times at higher microgel concentrations (Fig. 5a) are probably due to an increased size of the microgel aggregates. At a constant microgel concentration, the sizes of the aggregates do not change much when different amounts of naltrexone are added to the samples, and the lag time is therefore independent of the naltrexone concentration (Fig. 7a).

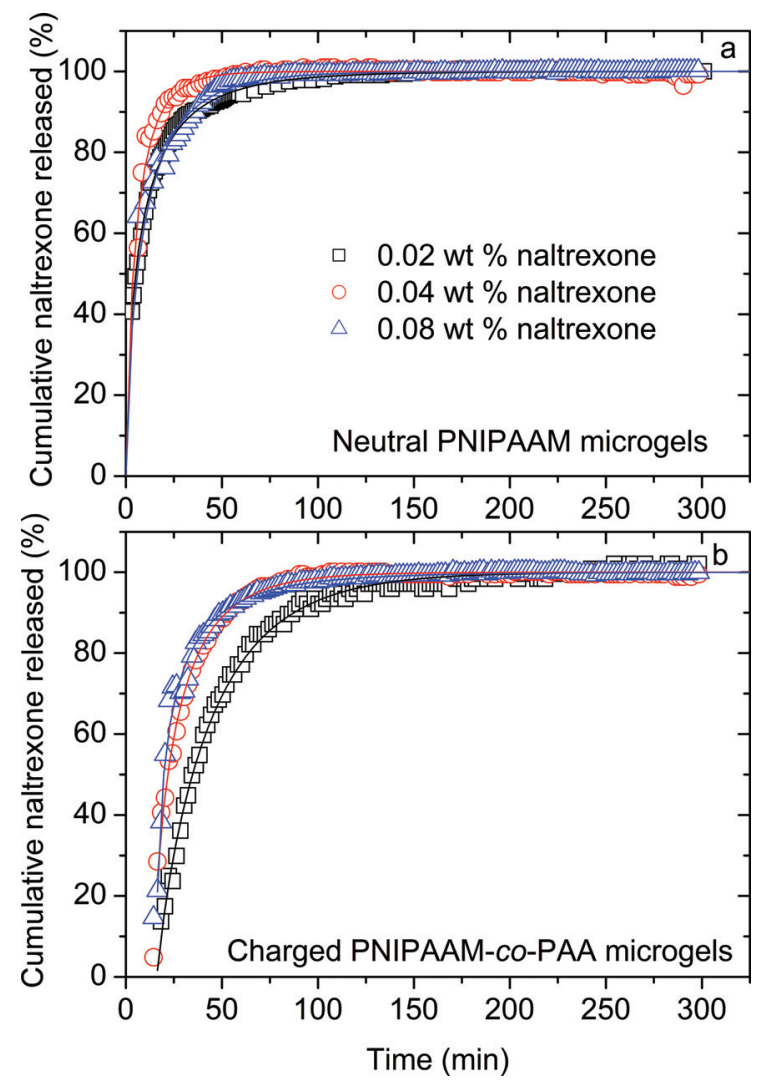

Figure 6. The effect of naltrexone concentration on the in vitro release profiles of naltrexone from $1.0 \mathrm{wt} \%$ of the two microgels in buffered suspensions $(\mathrm{pH} 7.4)$ at $37^{\circ} \mathrm{C}$. The lines are fitted with the aid of Eq. (2).

As can be seen from Figure 7b, for the charged microgels the release of the drug is faster when the drug loading is raised. However, for the neutral microgels there is no significant effect of naltrexone concentration on the release rates. At the highest naltrexone concentration, the release times for the two microgels are quite close to each other. This suggests that the faster release rates for the charged microgels at higher naltrexone concentrations might be due to a reduction of available charged groups on the microgels per naltrexone unit.

For the neutral microgels, the shape parameter, $\beta$, is not significantly affected by the naltrexone concentration. However, for the charged microgels, the value of $\beta$ decreases as the naltrexone concentration is raised (see Fig. 7c). It is possible that more of the charged PAA groups are associated with the oppositely charged drug at high naltrexone concentrations. Accordingly, the effective amount of repulsive forces within the microgels decreases. Without the repulsive forces, a more compact network is formed within the microgels (reminiscent of the neutral microgels). This is in agreement with Monte Carlo simulations, which predict a decrease of $\beta$ when the intermolecular distances within the matrix decreases. ${ }^{42}$

\section{CONCLUSIONS}

Poly( $N$-isopropylacrylamide) microgels without attached PAA chains and negatively charged PNIPAAM-co-PAA microgels were found to show sustained release of naltrexone. 


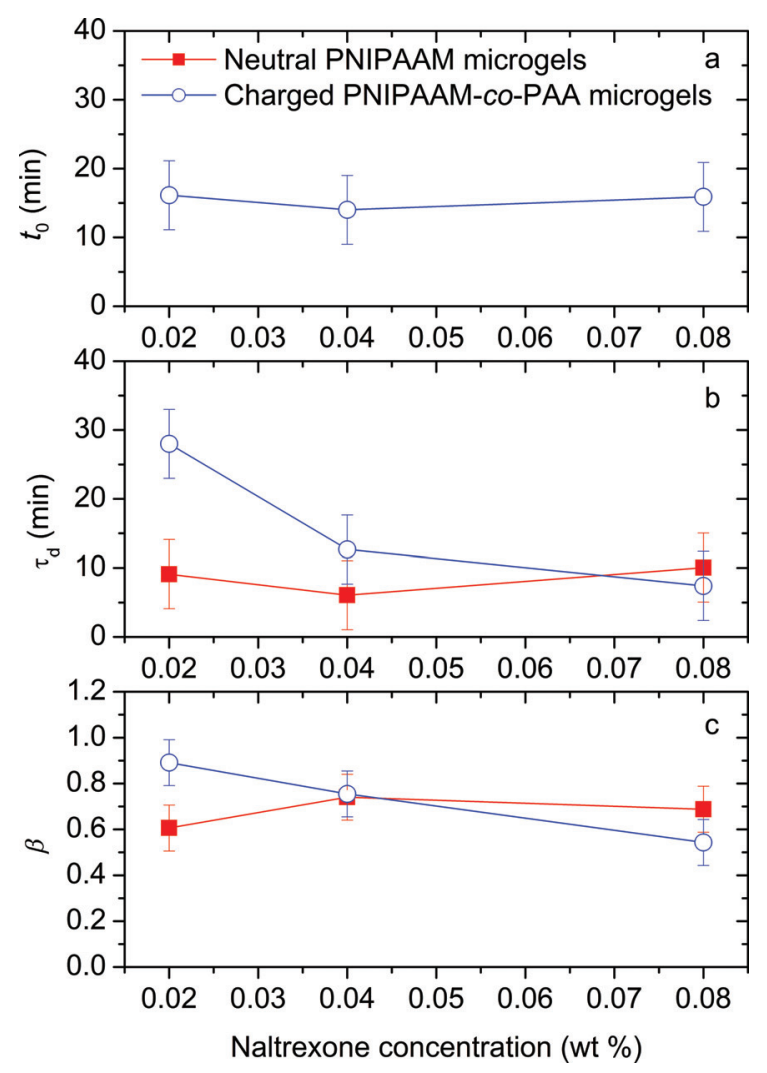

Figure 7. The effect of naltrexone concentration for the two microgels on (a) the lag time $\left(t_{0}\right)$, (b) the rate constant $\left(\tau_{\mathrm{d}}\right)$, and (c) the shape parameter $(\beta)$ for samples containing $1.0 \mathrm{wt} \%$ of the two microgels.

The release curves could successfully be represented by the Weibull equation.

Attractive associative interactions between naltrexone and oppositely charged microgels cause slower release times compared with the neutral microgels. The charges also cause the drug to diffuse further into the microgel aggregates, resulting in a release lag time.

Increasing the polymer concentration is expected to cause enhanced aggregation of the microgel suspensions. The resulting aggregates have a smaller specific leak surface, and accordingly the drug release times become longer. The larger aggregates also increase the release lag time of the charged microgels.

For the neutral microgels, the drug release is not significantly influenced by the naltrexone concentration. However, for the charged microgels both the drug release rates and the $\beta$ values are declining when the naltrexone content becomes higher. This might be caused by the reduced ratio between the charged groups on the microgels and the oppositely charged naltrexone. However, the amount of charged groups on the microgels is still high enough to prevent the release lag time from being affected by the naltrexone concentration.

The tested concentrations of the microgels did not exhibit any significant cytotoxic effect on a HeLa cells, making them promising candidates for drug delivery purposes. They also have the additional advantage of providing a system where the drug is easily loaded into the microgels, without any timeconsuming preparation procedures or the use of hash solvent conditions or severe mechanical stress.

\section{ACKNOWLEDGMENT}

Authors gratefully acknowledge financial support from the Norwegian Research Council for the project 190403.

\section{REFERENCES}

1. Preston KL, Silverman K, Umbricht A, DeJesus A, Montoya ID, Schuster CR. 1999. Improvement in naltrexone treatment compliance with contingency management. Drug Alcohol Depend 54:127-135.

2. Ferrari A, Bertolotti M, Dell'Utri A, Avico U, Sternieri E. 1998. Serum time course of naltrexone and $6 \beta$-naltrexol levels during long term treatment in drug addicts. Drug Alcohol Depend 52:211-220.

3. Kogan MJ, Verebey K, Mule SJ. 1977. Estimation of the systemic availability and other pharmacokinetic parameters of naltrexone in man after acute and chronic oral administration. Res Commun Chem Pathol Pharmacol 18:29-34.

4. Dinarvand R, Moghadam S, Mohammadyari-Fard L, Atyabi F. 2003. Preparation of biodegradable microspheres and matrix devices containing naltrexone. AAPS Pharm Sci Tech 4:45-54.

5. Liu Y, Sunderland VB, O'Neil AG. 2006. In vitro and in vivo release of naltrexone from biodegradable depot systems. Drug Dev Ind Pharm 32:85-94.

6. He GA, Hou HM, Liu XJ. 2001. Naltrexone microspheres: In vitro release and effect on morphine analgesia in mice. Acta Pharmacol Sin 22:530-533.

7. Akala EO, Wiriyacoonkasem P, Pan G. 2011. Studies on in vitro availability, degradation, and thermal properties of naltrexone-loaded biodegradable microspheres. Drug Dev Ind Pharm 37:673-684.

8. Yin W, Akala EO, Taylor RE. 2002. Design of naltrexone-loaded hydrolyzable crosslinked nanoparticles. Int J Pharm 244:9-19.

9. Salehi R, Davaran S, Rashidi MR, Entezami AA. 2009. Thermosensitive nanoparticles prepared from poly $(\mathrm{N}$-isopropylacrylamideacrylamide-vinilpyrrolidone) and its blend with poly(lactide-coglycolide) for efficient drug delivery system. J Appl Polym Sci 111:19051910 .

10. Narayani R, Panduranga Rao K. 1996. Gelatin microsphere cocktails of different sizes for the controlled release of anticancer drugs. Int J Pharm 143:255-258.

11. Bezemer JM, Radersma R, Grijpma DW, Dijkstra PJ, van Blitterswijk CA, Feijen J. 2000. Microspheres for protein delivery prepared from amphiphilic multiblock copolymers: 2 . Modulation of release rate. J Control Release 67:249-260.

12. Berkland C, King M, Cox A, Kim K, Pack DW. 2002. Precise control of PLG microsphere size provides enhanced control of drug release rate. J Control Release 82:137-147.

13. Segale L, Albertini B, Giovannelli L, Pattarino F. 2008. Empirical kinetic model of propafenone release from hot air coating microparticles. Int J Pharm 353:124-130.

14. Taşdelen B, Kayaman-Apohan N, MisirlI Z, Güven O, Baysal BM. 2005. Preparation, characterization, and drug-release properties of poly(N-isopropylacrylamide) microspheres having poly(itaconic acid) graft chains. J Appl Polym Sci 97:1115-1124.

15. Fundueanu G, Constantin M, Ascenzi P. 2009. Poly(Nisopropylacrylamide-co-acrylamide) cross-linked thermoresponsive microspheres obtained from preformed polymers: Influence of the physicochemical characteristics of drugs on their release profiles. Acta Biomater 5:363-373.

16. Chouhan R, Bajpai AK. 2010. Release dynamics of ciprofloxacin from swellable nanocarriers of poly(2-hydroxyethyl methacrylate): An in vitro study. Nanomed Nanotechnol Biol Med 6:453-462.

17. Kim S, Kim J-H, Kim D. 2011. pH sensitive swelling and releasing behavior of nano-gels based on polyaspartamide graft copolymers. J Colloid Interface Sci 356:100-106.

18. Keawchaoon L, Yoksan R. 2011. Preparation, characterization and in vitro release study of carvacrol-loaded chitosan nanoparticles. Colloids Surf B Biointerfaces 84:163-171. 
19. Batycky RP, Hanes J, Langer R, Edwards DA. 1997. A theoretical model of erosion and macromolecular drug release from biodegrading microspheres. J Pharm Sci 86:1464-1477.

20. Sanoj Rejinold N, Muthunarayanan M, Divyarani VV, Sreerekha PR, Chennazhi KP, Nair SV, Tamura H, Jayakumar R. 2011. Curcumin-loaded biocompatible thermoresponsive polymeric nanoparticles for cancer drug delivery. J Colloid Interface Sci 360:39-51.

21. Sanoj Rejinold N, Sreerekha PR, Chennazhi KP, Nair SV, Jayakumar R. 2011. Biocompatible, biodegradable and thermo-sensitive chitosan-g-poly (N-isopropylacrylamide) nanocarrier for curcumin drug delivery. Int J Biol Macromol 49:161-172.

22. Kim I-S, Jeong Y-I, Cho C-S, Kim S-H. 2000. Core-shell type polymeric nanoparticles composed of poly(l-lactic acid) and poly $(\mathrm{N}$ isopropylacrylamide). Int J Pharm 211:1-8.

23. Karewicz A, Zasada K, Szczubiałka K, Zapotoczny S, Lach R, Nowakowska M. 2010. "Smart" alginate-hydroxypropylcellulose microbeads for controlled release of heparin. Int J Pharm 385:163-169.

24. Vihola H, Laukkanen A, Hirvonen J, Tenhu H. 2002. Binding and release of drugs into and from thermosensitive poly(N-vinyl caprolactam) nanoparticles. Eur J Pharm Sci 16:69-74.

25. Reddy CLN, Swamy BY, Prasad CV, Aswini C, Mamatha P, Subha MCS, Rao KC. 2011. Synthesis and characterization of poly(NIPAMco-caprolactam) thermoresponsive micro-spheres for controlled release of acebutolol hydrochloride. Int J Pharm Pharm Sci 3:215-221.

26. Na K, Jung J, Lee J, Hyun J. 2010. Thermoresponsive pore structure of biopolymer microspheres for a smart drug carrier. Langmuir 26:11165-11169.

27. Constantin M, Cristera M, Ascenzi P, Fundueanu G. 2011. Lower critical solution temperature versus volume phase transition temperature in thermoresponsive drug delivery systems. Express Polym Lett 5:839-848.

28. Peng C-L, Tsai H-M, Yang S-J, Luo T-Y, Lin C-F, Lin W-J, Shieh MJ. 2011. Development of thermosensitive poly(n-isopropylacrylamideco-((2-dimethylamino) ethyl methacrylate))-based nanoparticles for controlled drug release. Nanotechnology 22:265608.

29. Al-Manasir N, Zhu K, Kjøniksen AL, Knudsen KD, Karlsson G, Nyström B. 2009. Effects of temperature and $\mathrm{pH}$ on the contraction and aggregation of microgels in aqueous suspensions. J Phys Chem B 113:11115-11123.

30. Kaufman JJ, Semo NM, Koski WS. 1975. Microelectrometric titration measurement of the pKa's and partition and drug distribution coefficients of narcotics and narcotic antagonists and their $\mathrm{pH}$ and temperature dependence. J Med Chem 18:647-655.

31. O'Brien J, Wilson I, Orton T, Pognan F. 2000. Investigation of the Alamar Blue (resazurin) fluorescent dye for the assessment of mammalian cell cytotoxicity. Eur J Biochem 267:5421-5426.

32. Zhang GH, Vadino WA, Yang TT, Cho WP, Chhaudry IA. 1994. Evaluation of the flow-through cell dissolution apparatus: Effects of flow rate, glass beads and tablet position on drug release from different type of tablets. Drug Dev Ind Pharm 20:2063-2078.

33. Iyer SS, Barr WH, Karnes HT. 2007. A 'biorelevant' approach to accelerated in vitro drug release testing of a biodegradable, naltrexone implant. Int J Pharm 340:119-125.

34. Weibull W. 1951. A statistical distribution function of wide applicability. J Appl Mech 18:293-297.

35. Lagenbucher F. 1976. Parametric representation of dissolution-rate curves by the RRSBW distribution. Die Pharm Industie 38:472-477.

36. Papadopoulou V, Kosmidis K, Vlachou M, Macheras P. 2006. On the use of the Weibull function for the discernment of drug release mechanisms. Int J Pharm 309:44-50.

37. Barzegar-Jalali M, Adibkia K, Valizadeh H, Shadbad MRS, Nokhodchi A, Omidi Y, Mohammadi G, Nezhadi SH, Hasan M. 2008. Kinetic analysis of drug release from nanoparticles. J Pharm Pharm Sci 11:167-177.
38. Kakran M, Sahoo NG, Li L, Judeh Z. 2010. Dissolution of artemisinin/polymer composite nanoparticles fabricated by evaporative precipitation of nanosuspension. J Pharm Pharmacol 62:413-421.

39. Aksungur P, Demirbilek M, Denkbas EB, Vandervoort J, Ludwig A, Unlu N. 2011. Development and characterization of cyclosporine A loaded nanoparticles for ocular drug delivery: Cellular toxicity, uptake, and kinetic studies. J Control Release 151:286-294.

40. Sahoo NG, Kakran M, Li L, Judeh Z, Muller RH. 2011. Dissolution enhancement of a poorly water-soluble antimalarial drug by means of a modified multi-fluid nozzle pilot spray drier. Mater Sci Eng C Mater Biol Appl 31:391-399.

41. Kosmidis K, Argyrakis P, Macheras P. 2003. Fractal kinetics in drug release from finite fractal matrices. J Chem Phys 119:6373-6377. 42. Kosmidis K, Argyrakis P, Macheras P. 2003. A reappraisal of drug release laws using Monte Carlo simulations: The prevalence of the Weibull function. Pharm Res 20:988-995.

43. Ignjatovic NL, Ninkov P, Sabetrasekh R, Uskokovic DP. 2010. A novel nano drug delivery system based on tigecycline-loaded calcium phosphate coated with poly-DL-lactide-co-glycolide. J Mater Sci Mater Med 21:231-239.

44. Wadajkar AS, Koppolu B, Rahimi M, Nguyen KT. 2009. Cytotoxic evaluation of $\mathrm{N}$-isopropylacrylamide monomers and temperaturesensitive poly(N-isopropylacrylamide) nanoparticles. J Nanopart Res 11:1375-1382.

45. Vega-Avila E, Pugsley MK. 2011. An overview of colorimetric assay methods used to assess survival or proliferation of mammalian cells. Proc West Pharmacol Soc 54:10-14.

46. Zhang XK, Meng LJ, Lu QH. 2009. Cell behaviors on polysaccharide-wrapped single-wall carbon nanotubes: A quantitative study of the surface properties of biomimetic nanofibrous scaffolds. ACS Nano 3:3200-3206.

47. Zhu XH, Tabata Y, Wang CH, Tong YW. 2008. Delivery of basic fibroblast growth factor from gelatin microsphere scaffold for the growth of human umbilical vein endothelial cells. Tissue Eng Part A 14:19391947.

48. Hasan AS, Socha M, Lamprecht A, El Ghazouani F, Sapin A, Hoffman A, Maincent P, Ubrich N. 2007. Effect of the microencapsulation of nanoparticles on the reduction of burst release. Int J Pharm 344:53-61.

49. Pignatello R, Musumeci T, Impallomeni G, Carnemolla GM, Puglisi G, Ballistreric A. 2009. Poly(3-hydroxybutyrate-coepsilon-caprolactone) copolymers and poly(3-hydroxybutyrate-co3-hydroxyvalerate-co-epsilon-caprolactone) terpolymers as novel materials for colloidal drug delivery systems. Eur J Pharm Sci 37:451-462.

50. Banquy X, Suarez F, Argaw A, Rabanel JM, Grutter P, Bouchard JF, Hildgen P, Giasson S. 2009. Effect of mechanical properties of hydrogel nanoparticles on macrophage cell uptake. Soft Matter 5:39843991.

51. Liu WJ, Chen HY, Zhou Y, Huang YM, Liu HL, Hu Y. 2009. Electrical properties and stability of poly(N-isopropylacylamide-comethacrylic acid) core-shell microgel. J Dispersion Sci Technol 30:1281-1287.

52. Jonassen H, Kjoniksen AL, Hiorth M. 2012. Effects of ionic strength on the size and compactness of chitosan nanoparticles. Colloid Polym Sci 290:919-929.

53. Tsianou M, Kjøniksen A, Thuresson K, Nyström B. 1999. Light scattering and viscoelasticity in aqueous mixtures of oppositely charged and hydrophobically modified polyelectrolytes. Macromolecules 32:29742982.

54. Baldursdóttir S, Kjøniksen A, Nyström B. 2006. The effect of riboflavin-photoinduced degradation of alginate matrices on the diffusion of poly(oxyethylene) probes in the polymer network. Eur Polym J 42:3050-3058. 